\title{
Interaction of sample dimension and sample-comparison mapping on pigeons' performance of delayed conditional discriminations
}

\author{
THOMAS R. ZENTALL \\ University of Kentucky, Lexington, Kentucky \\ PETER J. URCUIOLI \\ Purdue University, West Lafayette, Indiana \\ and \\ JOYCE A. JAGIELO and PAMELA JACKSON-SMITH \\ University of Kentucky, Lexington, Kentucky
}

\begin{abstract}
Coding strategies developed in the acquisition of delayed conditional discriminations can be assessed by independently manipulating sample and comparison memory load. Two stimulus dimensions that can affect memory load were examined: Number of stimuli in the sample and comparison sets (two vs. four) was manipulated between groups in a $2 \times 2$ design, and discriminability of sample and comparison stimuli (hues vs. lines) was manipulated between counterbalancing subgroups and within subjects. The results indicated large effects of sample discriminability but not of comparison discriminability, evidence for retrospective coding. There was also a significant effect of number of stimuli in the comparison set (although only with hard-to-discriminate samples) but not of number of stimuli in the sample set, evidence for prospective coding. These findings suggest evidence for retrospective coding with easy-to-discriminate samples, independently of number of stimuli in the comparison set, and evidence for prospective coding with hard-todiscriminate samples.
\end{abstract}

Research in animal memory has recently focused on the identification of the events that are represented in memory during the retention interval. For example, after having learned the contingencies between the samples and comparisons in delayed matching-to-sample (DMTS), a subject can perform correctly on the retention test by remembering the sample it saw at the beginning of the trial or, alternatively, which comparison stimulus to respond to after the retention interval. In other words, either retrospective coding of the samples or prospective coding of the comparisons can mediate delayed matching performances (Honig \& Thompson, 1982; Wasserman, 1986).

A considerable body of literature developed over the last few years appears to have established retrospective and prospective coding as distinct, separable memory processes (e.g., Capaldi, Nawrocki, \& Verry, 1983; J. S. Cohen, Galgan, \& Fuerst, 1986; Cook, Brown, \& Riley, 1985; Honig, Matheson, \& Dodd, 1984; Honig \& Wasserman, 1981; Peterson, 1984; Roitblat, 1980; Urcuioli \& Zentall, 1986; Zentall, Jagielo, Jackson-Smith, \& Urcuioli,

This research was supported by NSF Grant BNS-8418275 to Thomas R. Zentall and Peter J. Urcuioli. Correspondence concerning this article may be addressed to Thomas R. Zentall, Department of Psychology, University of Kentucky, Lexington, KY 40506.
1987). Less well established, however, are the conditions that affect which particular stimulus or response event(s) animals will code. Some recent studies (e.g., Honig \& Dodd, 1986), however, have begun to provide data relevant to the issue, and hypotheses regarding potentially influential variables have been offered. One hypothesis is that animals will preferentially code trial events either retrospectively or prospectively depending upon which results in the lesser amount of information to be remembered.

One way to manipulate memory load is to vary the number of stimuli in the sample and comparison sets. Santi and Roberts (1985) compared pigeons' memory performances on many-to-one and one-to-many DMTS. In their many-to-one procedure, only a single sample was presented on each trial, but, over trials, more than one sample was associated with (mapped onto) each correct comparison. In the one-to-many task, individual samples were associated with more than one correct comparison. Santi and Roberts reasoned that if pigeons prospectively code in DMTS, accuracy on many-to-one matching should be superior to that on one-to-many matching, because memory load would be relatively less on each trial in the former task than it would be on each trial in the latter. Conversely, if pigeons retrospectively code the samples in both tasks, memory performances should be comparable, because only 
one event (the sample) would be coded and remembered on each trial. Their finding that pigeons were more accurate on many-to-one than they were on one-to-many DMTS is thus consistent with a prospective coding interpretation.

Zentall, Jagielo, Jackson-Smith, and Urcuioli (1987) also examined the effects of sample-comparison mapping on pigeons' DMTS by varying the number of possible sample stimuli (two or four) and comparison stimuli (two or four) factorially across groups in two-alternative DMTS. Group performances during retention tests were affected only by the number of comparisons: Birds matching with two comparisons were more accurate over longer delays than were birds matching with four comparisons, but the number of sample stimuli did not affect performances. This result is similar to Santi and Roberts's (1985) finding that with fewer comparison alternatives, better DMTS performance is found, as is expected when birds code prospectively.

A second variable that has been manipulated in an attempt to identify pigeons' coding strategies is the discriminability of the sample and comparison sets. One might expect that manipulations of number and discriminability of stimuli would have comparable effects on retention gradients; however, evidence for retrospective coding has been found when the discriminability of the samples and comparisons is manipulated (i.e., performance on trials with hard-to-discriminate samples is significantly worse than is performance on trials with easyto-discriminate samples, but the discriminability of the comparisons has little or no effect on performance level; Urcuioli \& Zentall, 1986).

Can it be that these two manipulations evoke different coding processes in pigeons? And if so, is there some underlying principle that can account for these differences? If the differences in performance that result from manipulation of these two variables can be resolved, it would not only clarify the role played by number and discriminability of sample and comparison stimuli but it would also suggest that pigeons have considerable flexibility in their use of coding strategies.

The purpose of the present experiment was to integrate the results of Urcuioli and Zentall's (1986) study with those of Zentall et al.'s (1987) study. One potentially important difference between the two studies is that lines and shapes were used as the sample and/or comparison stimuli by Zentall et al. (1987), whereas hues and lines were used by Urcuioli and Zentall (1986). Perhaps the presence of highly discriminable hue sample stimuli in DMTS biases birds toward retrospective coding. In the present experiment, we manipulated both the number of stimuli in the sample and comparison sets and the discriminability of the samples and comparisons. Following the design of Zentall et al.'s (1987) experiment, four major groups were included in the present experiment to assess the effects of number of stimuli: One group was trained with two possible samples and two comparisons (2-2), another with four possible samples and two comparisons (4-2), a third with two possible samples and two possible pairs of comparisons (2-4), and the fourth with four possible samples and two possible pairs of comparisons (4-4). To assess the effects of stimulus discriminability, the stimuli used in the present experiment were highly discriminable hues (red and green) and less discriminable lines (vertical and horizontal), and withingroup comparisons were made (either between counterbalancing subgroups or within subjects, depending on the group and the comparison being made).

\section{METHOD}

\section{Subjects}

Thirty-two experimentally naive, 5-8 year-old White Carneaux pigeons (obtained from the Palmetto Pigeon Plant, Sumter, SC) served as subjects. Each pigeon was deprived to and maintained at $75 \%-80 \%$ of its free-feeding body weight during the experiment. Water and grit were continuously available in the home cages. The pigeons were maintained on a 12:12-h light:dark cycle in the colony room.

\section{Apparatus}

The experiment was conducted in a Lehigh Valley Electronics pigeon test chamber, with interior dimensions of $30 \times 35 \times 35 \mathrm{~cm}$ $(1 \times w \times h)$. Three horizontally aligned rectangular pecking keys $(3.2 \mathrm{~cm}$ wide $\times 2.5 \mathrm{~cm}$ high) were centered on the intelligence panel. The bottom edges of the keys were $16.0 \mathrm{~cm}$ above the chamber floor. Each key could be back-illuminated (by an Industrial Electronics Engineering inline projector equipped with G.E. \#1820 lamps) with red and green fields (Kodak Wratten filters Nos. 26 and 60 , respectively) and four vertical or horizontal white lines on a black background. Individual lines in the vertical and horizontal displays were $18 \mathrm{~mm}$ long $\times 3 \mathrm{~mm}$ wide and were separated from one another by $3 \mathrm{~mm}$. The opening to a rear-mounted grain feeder was located midway between the chamber floor and the bottom edge of the center response key. A shielded houselight provided dim illumination in the chamber during intertrial intervals. White noise and an exhaust fan attached to the chamber masked extraneous noises. Reinforcement consisted of 2-sec access to Purina Pigeon Grains. The experiment was controlled by solid-state equipment located in an adjoining room.

\section{Procedure}

Preliminary training. All pigeons were initially trained to eat from the grain feeder as soon as it was raised. Pecking a white center key was then shaped by the method of successive approximations, after which the center-key response requirement for reinforcement was gradually increased to 10 over the next four sessions.

Matching-to-sample training. On the day following the end of preliminary training, all birds began zero-delay, two-choice conditional discrimination training. For all subjects, each trial began with the presentation of a sample stimulus on the center key. Ten responses to the sample turned it off and immediately turned on the two comparison stimuli on the adjacent side keys. A single response to the correct comparison (see Table 1) turned off both comparisons, produced reinforcement, and then started a 10 -sec intertrial interval (ITI). A single response to the incorrect comparison simply turned off the comparisons and started the ITI. Each training session consisted of 96 trials.

Birds in the 2-2 group matched two samples to the same two comparison stimuli. The samples and comparisons were red $(R)$ and green (G) hues for half of the birds, and vertical (V) and horizontal (H) lines for the other half. For pigeons assigned to the 2-4 group, the matching task involved two samples and, on each trial, one of two possible pairs of comparison stimuli (either $R$ and $G$ hues or 
Table 1

Trial Types for the Four Groups

\begin{tabular}{|c|c|c|c|}
\hline $2-2$ & $2-4$ & $4-2$ & $4-4$ \\
\hline $\begin{array}{l}R(R, G) \\
G(G, R)\end{array}$ & $\begin{array}{ll}R & (R, G) \\
G & (G, R) \\
R & (V, H) \\
G & (H, V)\end{array}$ & $\begin{array}{l}R \quad(R, G) \\
G \quad(G, R) \\
V \quad(R, G) \\
H \quad(G, R)\end{array}$ & $\begin{array}{l}R(\mathrm{R}, \mathrm{G}) \\
\mathrm{G}(\mathrm{G}, \mathrm{R}) \\
\mathrm{V}(\mathrm{V}, \mathrm{H}) \\
\mathrm{H}(\mathrm{H}, \mathrm{V})\end{array}$ \\
\hline \multicolumn{4}{|c|}{ or } \\
\hline $\begin{array}{l}V(\mathbf{V}, \mathbf{H}) \\
\mathrm{H}(\mathbf{H}, \mathbf{V})\end{array}$ & $\begin{array}{l}V(V, H) \\
H \quad(H, V) \\
V(R, G) \\
H \quad(G, R)\end{array}$ & $\begin{array}{l}V(V, H) \\
H \quad(H, V) \\
R(V, H) \\
G(H, V)\end{array}$ & $\begin{array}{l}V(V, H) \\
H(H, V) \\
R(R, G) \\
G(G, R)\end{array}$ \\
\hline
\end{tabular}

Note- $\mathrm{V}=$ vertical lines, $\mathrm{H}=$ horizontal lines, $\mathrm{R}=\mathrm{red}, \mathrm{G}=$ green. The first letter represents the samples stimulus, and the two letters in parentheses represent the comparison stimuli. The first letter in the parentheses is the correct comparison for that trial type.

Table 2

Average Number of Sessions to $90 \%$ Accuracy During Zero-Delay Matching Acquisition

\begin{tabular}{crrrr}
\hline & \multicolumn{4}{c}{ Trial Type } \\
\cline { 2 - 5 } Group & H-H & H-L & L-H & L-L \\
\hline $2-2$ & 17.5 & & & 18.0 \\
$2-4$ & 22.8 & 30.0 & 27.2 & 32.8 \\
$4-2$ & 19.5 & 21.0 & 52.0 & 30.0 \\
$4-4$ & 21.2 & & & 47.8 \\
\hline Note-H & &
\end{tabular}

$V$ and $H$ lines). The samples were $R$ and $G$ for half of the $2-4$ birds, and $\mathrm{V}$ and $\mathrm{H}$ for the other half. Birds in the 4-2 group had four possible samples $(R, G, V$, or $H)$ but only one pair of comparison stimuli. The comparisons were $R$ and $G$ for half of the birds in this group, and $\mathrm{V}$ and $\mathrm{H}$ for the remaining birds. Finally, birds in the 4-4 group matched all four individual stimuli ( $R, G, V$, and $H)$ to each other. Hue comparisons were always presented on hue-sample trials, and line comparisons on line-sample trials.

Trials within each session were counterbalanced for sample stimulus, comparison stimulus pair (where appropriate), and the left-right location of individual comparisons. On trials involving sample and comparison stimuli from the same dimension, the correct comparison was the one that physically matched the sample stimulus. On trials involving samples and comparisons from different dimensions, $\mathrm{V}$ was correct following the $\mathrm{R}$ sample, $\mathrm{H}$ was correct following the $G$ sample, and vice versa. The trial types for each group are summarized in Table 1.

The experiment was run in two replications of 16 birds each. For each replication, 4 birds were assigned randomly to each of the four groups described above. The birds received a total of 100 and 70 sessions of training, respectively, in the first and second replications.

Delayed matching-to-sample testing. Following acquisition of the zero-delay task, all birds were tested for 50 96-trial sessions on DMTS in which delays of $0,1,2$, and 4 sec occurred randomly between offset of the sample and onset of the comparisons. In each DMTS session, each of the four delays occurred equally often with each of the groups' respective trial types. All other aspects of the procedure were identical to those described previously for matchingto-sample training.

\section{RESULTS}

\section{Zero-Delay Matching}

One bird in the first replication failed to reach criterion during zero-delay acquisition and was not tested on DMTS. For the analysis of the acquisition data, this bird was given a score of 101 sessions-to-criterion.
Acquisition of zero-delay matching was faster for groups matching only two samples than it was for groups matching four. These results can be seen in Figure 1, which depicts the average number of sessions for each group to reach a $90 \%$ level of accuracy.

A three-way ANOVA performed on the acquisition data, with number of samples, number of comparisons, and replications as factors, showed a significant effect of number of samples $[F(1,24)=6.21]$. Neither the effect of number of comparisons $[F(1,24)=1.65]$ nor the interaction between number of samples and number of comparisons $(F<1)$ was significant, however. The only other significant effect was due to replications: Birds in the second replication learned faster than did birds in the first $[F(1,24)=5.03]$. However, since this factor did not interact significantly with any other variable, it was ignored in all subsequent analyses.

Separate ANOVAs were then performed on the data from each group to analyze stimulus effects (hues vs. lines) across the various subgroups and subconditions during acquisition. These data are shown in Table 2 . In the 2-2 group, acquisition by the 4 birds matching $R$ and $G$ hues was not significantly different from acquisition by the 4 birds matching $\mathrm{V}$ and $\mathrm{H}$ lines $(F<1)$. Similarly, the 4 birds in the $2-4$ group that matched hue samples did not acquire their task significantly faster than did the birds that matched line samples $(F<1)$. Although there appeared to be a within-subject effect of comparison dimension in the 2-4 group (suggesting that matching on huecomparison trials was learned faster than was matching on line-comparison trials), this difference was not quite significant $[F(1,6)=4.38]$. In the $4-2$ group, matching on hue-sample trials appeared to be learned more rapidly than did matching on line-sample trials; however, this within-subject effect of sample dimension did not quite reach statistical significance $[F(1,6)=5.19]$. The 4 birds trained with hue comparisons learned zero-delay matching at about the same rate as did those trained with line comparisons $(F<1)$. Finally, the birds in the $4-4$ group did learn to match more quickly with hue samples than

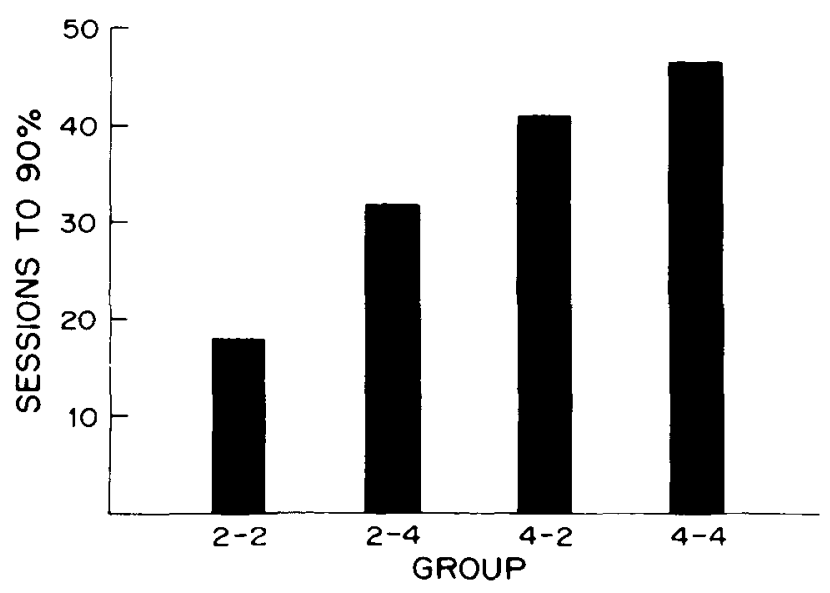

Figure 1. Mean number of sessions to $90 \%$ accuracy during acquisition by each group. 
they did with line samples and comparisons $[F(1,7)=$ 11.09]. If one pools data from the two groups for which sample and comparison dimension could be examined independently (i.e., the 2-4 and 4-2 groups), acquisition with hue samples was faster ( $M=23.3$ sessions) than was acquisition with line samples ( $M=35.5$ sessions), but acquisition with hue comparisons was comparable $(M=30.4$ sessions) to that with line comparisons $(M=28.4$ sessions).

\section{DMTS Testing}

The average retention gradient for each group pooled over the 50 sessions of DMTS is plotted in Figure 2. These data suggest that the two groups matching with only two comparison stimuli (viz., the 2-2 and 4-2 groups) were more accurate over longer delays than were the two groups matching with four comparisons (viz., the 2-4 and 4-4 groups). A two-way ANOVA on the group data, however, failed to reveal a significant effect of number of comparison stimuli $[F(1,27)=1.51]$. Additionally, neither the effect of number of samples nor the samples $\times$ comparisons interaction were significant (both $F \mathrm{~s}<1$ ). Inspection of the data within each group indicated that large within-group variability due to sample dimension was responsible for the absence of statistically significant findings.

Figure 3 shows the effect of stimulus dimension in this experiment, with DMTS accuracy plotted separately for each subgroup in the 2-2, 2-4, and 4-2 groups, and for the hue versus line within-subject subconditions in the 2-4, 4-2, and 4-4 groups. As can be seen in each panel of this figure, performances on trials involving $R$ and $G$ hues as samples were consistently more accurate than were performances on trials with $\mathrm{V}$ and $\mathrm{H}$ line samples.

This result was confirmed by separate ANOVAs on the data from each group. Analysis of the retention data from the 2-2 group showed a significant effect of stimulus dimension $[F(1,6)=9.17]$, delay interval $[F(3,18)=29.97]$, and stimulus $\times$ delay interaction $[F(3,18)=7.46]$. Similarly, analysis of the 2-4 group data indicated a signifi-

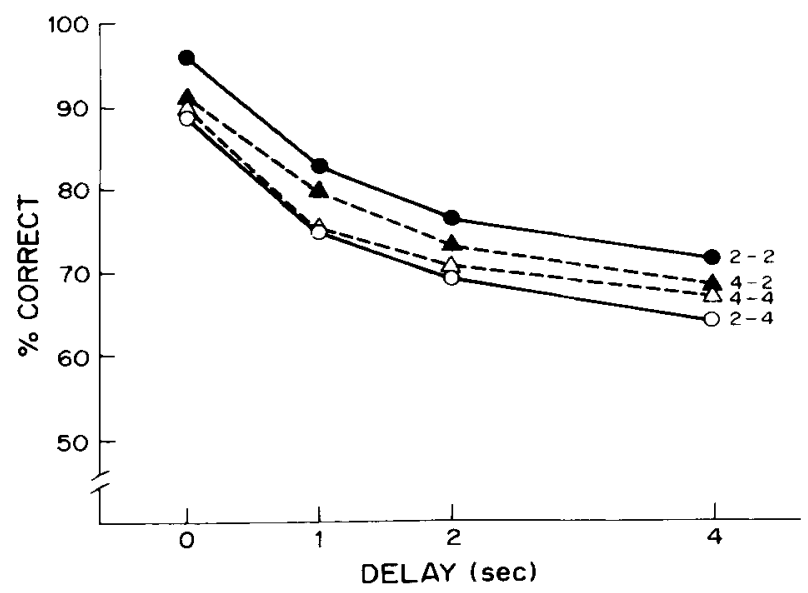

Figure 2. Retention gradients for each group pooled over the $\mathbf{5 0}$ delayed matching-to-sample test sessions.

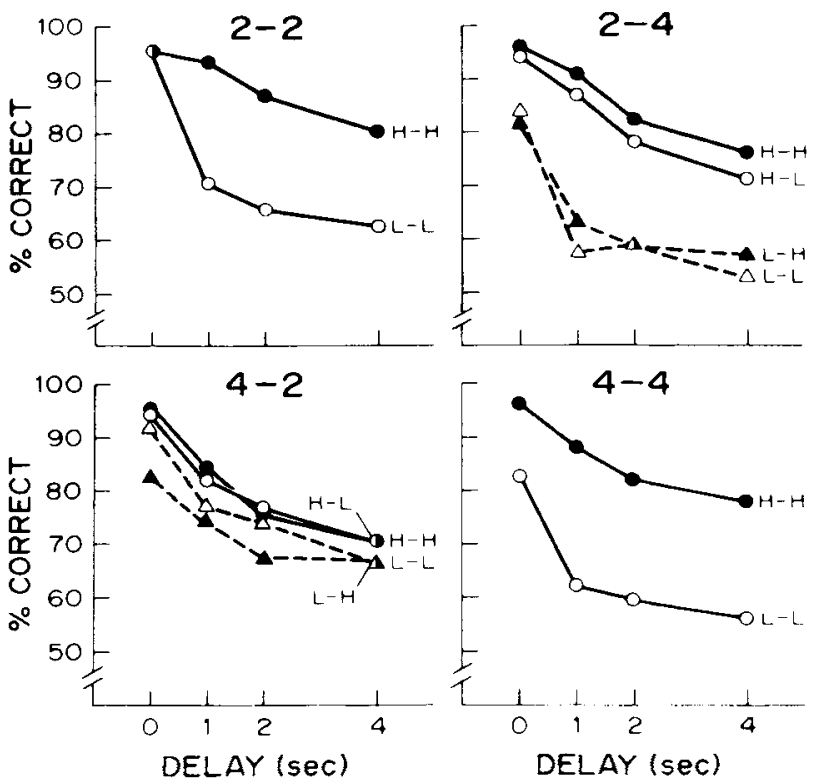

Figure 3. Retention gradients for each group on each trial type. The two-letter trial designations in each panel indicate type of sample ( $H=$ hues; $L=$ lines), followed by type of comparison ( $H$ or $L)$.

cant effect of sample dimension $[F(1,6)=11.45]$, delay interval $[F(3,18)=48.35]$, and sample $\times$ delay interaction $[F(3,18)=4.80]$. The within-subject effect of comparison dimension in this group, however, was not significant $[F(1,6)=1.79]$, nor was any other interaction.

Analysis of the retention data from the 4-2 group showed a significant within-subjects effect of sample dimension $[F(1,6)=14.37]$ and a significant effect of delay interval $[F(3,18)=27.23]$. The interaction between samples and delays was not significant, however $(F<1)$. As in the 2-4 group, the effect of comparison dimension was not significant $(F<1)$, nor was any other interaction. Finally, an ANOVA on the 4-4 data revealed a significant within-subjects effect of stimulus dimension $[F(1,6)=33.91]$, delay $[F(3,18)=55.78]$, and stimulus $\times$ delay interaction $[F(3,18)=3.85]$.

Although significant effects of stimulus dimension were found in all four groups, examination of Figure 3 reveals that the effect was smaller in the 4-2 group than it was in any of the other three. Statistical analysis involving direct comparison among these groups, however, is inappropriate because the stimulus-dimension variables are sometimes within subjects and sometimes between groups. Nonetheless, if one ignores comparison-dimension effects, which were minimal in both the 4-2 group (between subgroups) and the 4-4 group (within subjects), one can reasonably ask if the within-subjects, sample-stimulus dimension effect was smaller in the 4-2 group than it was in the 4-4 group. This analysis, involving group (4-2 vs. 4-4), sample dimension (hues vs. lines), and delay as factors, yielded a nonsignificant group effect $(F<1)$, but significant effects of sample dimension $[F(1,13)=45.34]$ and delay $[F(1,13)=74.23]$ were found. More impor- 
tantly, the analysis revealed a significant group $\times$ sampledimension interaction $[F(1,13)=15.84]$, indicating that the magnitude of the sample-dimension effect was indeed smaller in the 4-2 group than it was in the 4-4 group.

\section{DISCUSSION}

The acquisition data from this experiment support our previous findings that increasing the number of stimuli in the sample set slows the rate of MTS acquisition (Zentall et al., 1987). Similarly, although we have found that increasing the number of stimuli in the comparison set also slows the rate of MTS acquisition (Zentall et al., 1987), in the present experiment this difference did not reach statistical significance. This outcome was not unanticipated, however, because stimulus variables that affect acquisition of conditional discriminations tend to have a greater effect when samples, rather than comparisons, are manipulated. For example, Carter and Eckerman (1975) reported that the effects of sample discriminability on acquisition are substantially greater than the effects of comparison discriminability.

Within the 4-2, 2-4, and 4-4 groups of the present experiment, there were clear trends toward faster acquisition on trials involving hue stimuli than that on trials involving line stimuli. Somewhat surprisingly, though, matching acquisition with hue samples and comparisons in the 2-2 groups was no more rapid than it was with line samples and comparisons. The failure to replicate the often reported difference between these latter two conditions (Carter \& Eckerman, 1975; L. R. Cohen, Looney, Brady, \& Aucella, 1976; Urcuioli \& Zentall, 1986, Experiment 2) may be related to the relatively small number of pigeons per subgroup $(n=4)$ and, perhaps, to the fact that the line stimuli that were used (four white stripes on a black background) may have been somewhat more discriminable than were the single-line stimuli more typically used.

The DMTS test data appeared to support our previous findings that memory performance is poorer with four comparisons than it is with only two (Zentall et al., 1987), but the present effect was small and not statistically reliable. The failure to find a significant effect seems to have resulted, in large part, from the substantial within-group variability produced by the hue versus line stimulus difference. In all four groups, performances on trials with hue samples were considerably more accurate than were performances on trials with line samples, an effect consistent with that reported by Urcuioli and Zentall (1986).

The present findings thus confirm our suspicions that hue and line stimuli might produce somewhat different results from those reported by Zentall et al. (1987) with lines and shapes. Furthermore, the present results seem to lead to a paradoxical conclusion. The trend in the overall group data suggests that memory performance was better when the task involved two rather than four comparisons (no similar trend was evident as a function of the number of sample stimuli). When manipulations of comparison stimuli affect memory performance, but corresponding manipulations of sample stimuli do not, a prospective memory process is implicated (Roitblat, 1980).

On the other hand, memory performances within each group were more accurate with hue samples than they were with line samples, but performances were unaffected by similar variation in comparison dimension. When manipulations of sample stimuli affect memory, but corresponding manipulations of comparisons do not, a retrospective memory process is implicated.

One explanation for this paradox is that pigeons may treat hue- and line-sample trials quite differently. For example, birds may retrospectively code hue samples because they are particularly discriminable. With less discriminable line samples, they may prospectively code. If this explanation is correct, then reanalysis of the overall group test data, using only the line-sample trials, should reveal between-group differences. The results from this reanalysis are plotted in Figure 4. The figure shows a larger separation between the gradients from the two- and four-comparison groups than that shown in Figure 2. An ANOVA performed on these data confirmed that DMTS performances were more accurate with only two comparisons $(74.6 \%$ correct) than they were with four $(64.6 \%$ correct) $[F(1,19)=5.86]$. A similar analysis performed on the data from hue-sample trials alone (not shown) indicated no difference in performance: $85.1 \%$ correct for two-comparison tasks versus $85.2 \%$ for four-comparison tasks $(F<1)$.

Thus, it appears that the direction of coding is affected by the characteristics of the sample stimuli. When the samples are easy to discriminate (e.g., red and green hues), they may bias the pigeons to retrospectively code them independently of the number of comparison stimuli. When the samples are hard to discriminate (e.g., vertical and horizontal lines), however, they do not appear to have that same biasing effect and may even bias in the opposite direction, as revealed by greater sensitivity to the number of stimuli in the comparison set.

One unexpected finding was that the effect of sample dimension in the 4-2 group was smaller than it was in each

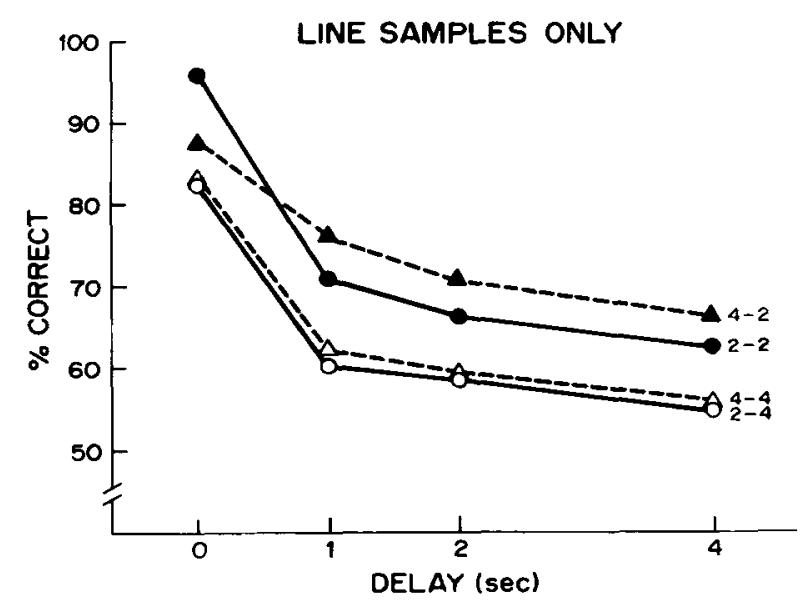

Figure 4. Retention gradients for line-sample trials for each group. 
of the other three groups. The 4-2 group was the only group for which hue and line samples were mapped onto the same correct comparison. This finding raises the possibility that samples associated with the same correct comparison are commonly coded (e.g., red and vertical samples are both coded as "Sample A" because they are both associated with a vertical comparison). If this hypothesis is correct, two predictions follow. First, if pigeons are trained on a many-to-one DMTS task in Phase 1 (e.g., red and vertical samples mapped onto vertical comparisons, and green and horizontal samples mapped onto horizontal comparisons) and, in Phase 2, one pair of samples (e.g., red and green) is associated with a new pair of comparisons (e.g., circle and dot), then, in Phase 3, when the remaining pair of samples (vertical and horizontal) is paired with the Phase 2 comparisons (circle and dot), one should find evidence for preexisting associations between never previously paired samples and comparisons. Second, if one examines intertrial-interference effects in a many-to-one task, one should find evidence for interference from the sample on the previous trial, if that sample is associated with the comparison that is incorrect on the present trial. And more importantly, one should find that the interference occurs whether the current sample and the prior sample are from the same dimension or from different dimensions, because, in both cases, the common codes elicited by the prior trial are incompatible with the correct comparison on the present trial. We recently have conducted experiments that provide evidence for both predictions (Urcuioli, Zentall, Jackson-Smith, \& Steirn, in press).

Finally, it is possible to make a comparison between the present findings and those of Santi and Roberts (1985), who reported that pigeons' performances on one-to-many DMTS were less accurate than were those on many-toone DMTS. Their data cannot be directly compared with ours, however, because Santi and Roberts did not counterbalance for stimulus dimension (i.e., both groups were trained with two-hue DMTS; lines and shapes were added to the sample set of their many-to-one group, and lines and shapes were added to the comparison set of their one-to-many group). In presenting their data, Santi and Roberts separately plotted data from hue-matching trials (all hues) and data from mixed hue/line-shape trials. In the case of the mixed trials, these data represented hue samples and line-shape comparisons for the one-to-many group and line-shape samples and hue comparisons for the many-to-one group. Corresponding data from the present experiment are plotted in Figure 5: $\mathrm{H}-\mathrm{L}$ performance of the 2-4 (one-to-many) group is shown in comparison to $\mathrm{L}-\mathrm{H}$ performance of the 4-2 (many-to-one) group. The difference between conditions observed here is opposite in direction to that reported by Santi and Roberts (1985), although an ANOVA performed on these data indicated that the difference was not significant $[F(1,7)=1.79]$. Similarly, H-H performance of the 2-4 (one-to-many) group is shown in comparison to $\mathrm{H}-\mathrm{H}$ performance of the 4-2 (many-to-one) group. Again, although the difference in conditions was quite small, it was opposite in direction to that reported by Santi and Roberts. Of course, the present data represent only a subset of the data from half of the pigeons in these two groups, so the power of detecting a true difference is undoubtedly very small. Nonetheless, our data are consistent with the other retention data obtained in this experiment (cf. Figure 3), and they are consistent with the overall interpretation we have proposed. Thus, our findings must at least question the reliability of Santi and Roberts's findings.

The results of the present experiment demonstrate some of the complexities associated with animal memory processes. Our findings suggest that highly discriminable sample stimuli (e.g., red and green hues) may encourage retrospective coding by pigeons, whereas less discriminable sample stimuli (e.g., vertical and horizontal lines) do not bias pigeons to retrospectively code the samples, but,

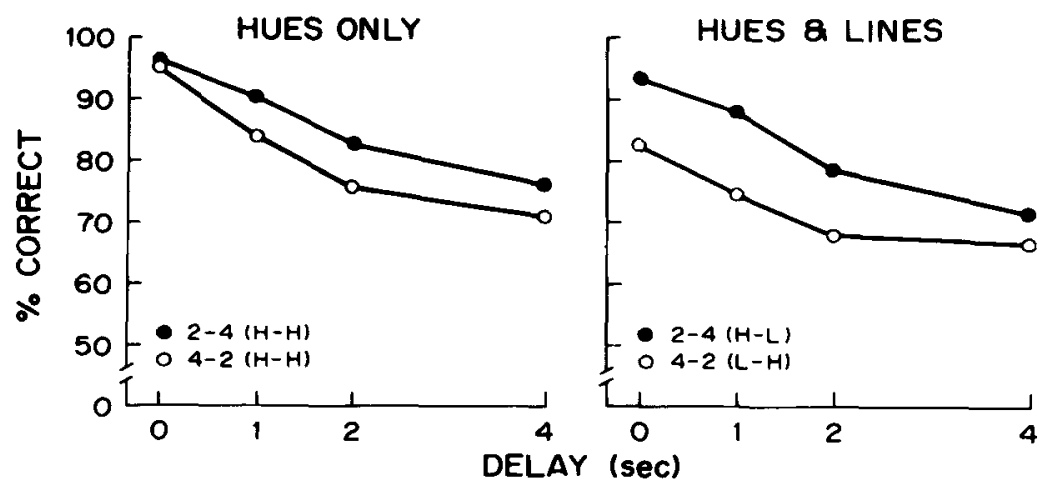

Figure 5. Retention gradients for Group 2-4 on hue sample-line comparison (H-L) trials and on hue sample-hue comparison (H-H) trials versus Group 4-2 on line sample-hue comparison (L-H) trials and on hue sample-hue comparison (H-H) trials. See text for further details. 
instead, permit greater sensitivity to the anticipated attributes of the comparison set (e.g., number of comparison stimuli).

\section{REFERENCES}

CAPAldi, E. J., NAWrocki, T. M., \& VerRY, D. R. (1983). The nature of anticipation: An inter- and intraevent process. Animal Learning \& Behavior, 11, 193-198.

CARTer, D. E., \& Eckerman, D. A. (1975). Symbolic matching by pigeons: Rate of learning complex discriminations predicted from simple discriminations. Science, 187, 662-664.

Cohen, J. S., Galgan, R., \& Fuerst, D. (1986). Retrospective and prospective short-term memory in delayed response tasks in rats. Animal Learning \& Behavior, 14, 38-50.

Cohen, L. R., LoOney, T. A., Brady, J. H., \& Aucella, A. F. (1976). Differential sample response schedules in the acquisition of conditional discriminations by pigeons. Joumal of the Experimental Analysis of Behavior, 26, 301-316.

Cook, R. G., Brown, M. F., \& Riley, D. A. (1985). Flexible memory processing by rats: Use of prospective and retrospective information in the radial maze. Journal of Experimental Psychology: Animal Behavior Processes, 11, 453-469.

HonIG, W. K., \& DoDD, P. W. D. (1986). Anticipation and intention in working memory. In D. F. Kendrick, M. E. Rilling, \& M. R. Denny (Eds.), Theories of animal memory (pp. 77-100). Hillsdale, NJ: Erlbaum.

Honig, W. K., Matheson, W. R., \& Dodd, P. W. D. (1984). Outcome expectancies as mediators for discriminative responding. Canadian Journal of Psychology, 38, 196-217.

Honig, W. K., \& Thompson, R. K. R. (1982). Retrospective and prospective processing in animal working memory. In G. H. Bower
(Ed.), The psychology of leaming and motivation (Vol. 16, pp. 167197). New York: Academic Press.

Honig, W. K., \& Wasserman, E. A. (1981). Performance of pigeons on delayed simple and conditional discriminations under equivalent training conditions. Learning \& Motivation, 12, 149-170.

Peterson, G. B. (1984). How expectancies guide behavior. In H. L. Roitblat, T. G. Bever, \& H. S. Terrace (Eds.), Animal cognition (pp. 135-147). Hillsdale, NJ: Erlbaum.

RoITBLAT, H. L. (1980). Codes and coding processes in pigeon shortterm memory. Animal Learning \& Behavior, 8, 341-351.

Santi, A., Roberts, W. A. (1985). Prospective representation: The effects of varied mapping of sample stimuli to comparison stimuli and differential trial outcomes on pigeons' working memory. Animal Leaming \& Behavior, 13, 103-108.

UrCUIOLI, P. J., \& ZeNTALl, T. R. (1986). Retrospective coding in pigeons' delayed matching-to-sample. Journal of Experimental Psychology: Animal Behavior Processes, 12, 69-77.

Urcuioli, P. J., Zentall, T. R., Jackson-Smith, P., \& Steirn, J. N. (in press). Evidence for common coding in many-to-one matching: Retention, intertrial interference, and transfer. Joumal of Experimental Psychology: Animal Behavior Processes.

Wasserman, E. A. (1986). Prospection and retrospection as processes of animal short-term memory. In D. F. Kendrick, M. E. Rilling, \& M. R. Denny (Eds.), Theories of animal memory (pp. 53-75). Hillsdale, NJ: Erlbaum.

Zentall, T. R., Jagielo, J. A., Jackson-Smith, P., \& Urculoli, P. J. (1987). Memory codes in pigeon short-term memory: Effects of varying the number of sample and comparison stimuli. Learning \& Motivation, 18, 21-33.

(Manuscript received June 28, 1988; revision accepted for publication October 14, 1988.) 